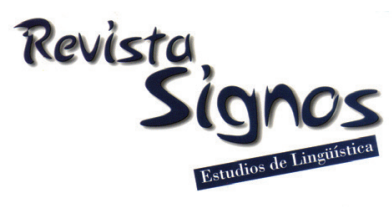

\title{
La construcción retórica del conflicto armado colombiano: Metáfora y legitimación del carácter bélico del conflicto ${ }^{1}$
}

\author{
The rhetorical construction of the Colombian armed conflict: \\ Metaphor and legitimization of warlike status of the conflict
}

\section{Giohanny Olave}

Universidad de Buenos Aires

ARGENTINA

olavearias@gmail.com

Recibido: 29-IX-2011 / Aceptado: 31-V-2012

\section{Resumen}

Se realiza una aproximación al conflicto armado colombiano reciente desde el análisis discursivo, a partir de una muestra de comunicados oficiales del Presidente de Colombia, Juan Manuel Santos, donde se hace referencia al combate del Ejército Estatal con la guerrilla de las "Fuerzas Armadas Revolucionarias de Colombia, Ejército del Pueblo" (Farc-Ep). El análisis se concentra en el problema de la dimensión argumentativa de los usos metafóricos en el ámbito político. Al caso seleccionado se le aplican dos modelos teóricos (uno cognitivo y el otro argumentativo) que confluyen en los resultados, evidenciando la búsqueda de legitimación del carácter bélico del conflicto por parte del actor que usa la metáfora. Se concluye que la metáfora conceptual establece un marco cognitivo dentro del cual la aceptabilidad, relevancia y adecuación de las premisas difícilmente son puestas en discusión por parte de la audiencia. Tanto el apoyo (la fuerza de las experiencias) como la garantía (la fuerza de los principios) se presentan como construcciones discursivas al servicio de la aceptabilidad de la pretensión, entendida como espacio conceptual integrado. Finalmente, se discute la lógica que subyace en la construcción conceptual instaurada a partir de la metáfora, y se proyecta la continuidad del estudio del conflicto armado desde el enfoque discursivo con orientación sociocognitiva.

Palabras Clave: Discurso, argumentación, metáfora conceptual, blending. 


\begin{abstract}
This paper presents an approach to the recent armed conflict in Colombia based on discourse analysis, from a corpus of official statements of the President of Colombia, Juan Manuel Santos, which refers to the State Army combat with the guerrillas of the "Fuerzas Armadas Revolucionarias de Colombia, Ejército del Pueblo, FARC-EP". The analysis focuses on the argumentative dimension of metaphorical uses in the political arena. Two theoretical models are applied to the case study: a cognitive model and an argumentative one, that converge on the results, highlighting the search for legitimacy the warlike status of the conflict, by the actor who uses the metaphor. We conclude that the conceptual metaphor provides a cognitive framework within which the acceptability, relevance and suitability of the premises are hardly taken up for discussion by the audience. Both the support (the strength of the experience) and the warrant (the force of the principles) are presented as discursive constructions that serve the acceptability of the claim, understood as an integrated conceptual space. Finally, we discuss the logic that underlies the construction instituted from conceptual metaphor, and we expect to continue the study of armed conflict based on the sociocognitive oriented discourse approach.
\end{abstract}

Key Words: Discourse, argumentation, conceptual metaphor, blending.

\title{
INTRODUCCIÓN
}

Los estudios más amplios sobre la guerra, la violencia y las guerrillas en Colombia suelen simplificar la cuestión del lenguaje aduciendo que la búsqueda de los principios ideológicos, políticos y económicos que las originan aporta más a la comprensión del conflicto o a la toma de decisiones inmediatas que coadyuven a frenar estos procesos.

Al referirse a la situación bélica en Colombia, Estrada (2004: 27) advierte que:

"la guerra y el conflicto armado se han institucionalizado, es decir, comprenden discursos regulares, espacios oficiales de difusión, medios de comunicación a su servicio, textos y formas de hablar de la guerra que ya hacen parte de la vida cotidiana”.

En el contexto de la violencia armada se ven involucrados los discursos que pretenden justificar la cara terrible del abuso físico que le es propio, con lo cual el comportamiento discursivo deviene en conducta política que convalida el accionar bélico a través de estrategias particulares.

El análisis de los discursos deslegitimadores de sus actores (Sabucedo, Barreto, Borja, De la Corte, Durán \& Blanco, 2004; Sabucedo, Barreto, Borja, De la Corte \& Durán, 2006; Sabucedo, Barreto, Borja \& López, 2008), el estudio de la acción política de las metáforas en el conflicto (Estrada, 2000, 2004; Posada Carbó, 2002, 2003, 2005, 2006), la delimitación de estrategias retóricas en uso por parte de los 
actores (Salamanca, 2006) y el interés en el análisis de los conceptos que se van construyendo en función de los intereses de los involucrados (Vizcaíno, 2004; Castro Caycedo \& CMP, 2005; Shlenker \& Iturralde, 2006; Gómez, 2009) hacen parte del espectro actual de estudios disponibles en torno a las relaciones entre discurso y conflicto, línea de trabajo en la que inscribo el análisis discursivo de la justificación del carácter bélico.

Un proyecto de descripción o de re-descripción de los hechos involucrados es una re-orientación del análisis de las cuestiones, centrada en el discurso, con el belicismo como objeto de discusión. El área de trabajo más amplia es la configuración de marcos de acción política sobre una retórica de la justificación del uso de las armas, área sobre la cual se cruzan procesos de conceptualización que construyen la significación del conflicto. El trabajo ubica su pertinencia en esta revisión de los significados que involucran, diseminan e imponen los actores del conflicto armado colombiano desde sus discursos, de manera que en la guerra el decir se concibe también como un hacer particularmente transformador: acciones cuya comprensión convoca el análisis discursivo desde la acción situada.

Concretamente, esta exposición se concentrará en indagar la dimensión argumentativa de una metáfora conceptual rastreada en los discursos del Presidente de Colombia, Juan Manuel Santos, en referencia directa a los actores armados de la guerrilla de las Fuerzas Armadas Revolucionarias de Colombia, Ejército del Pueblo (Farc-Ep).

\section{Marco de referencia}

Para tal indagación, se propone un marco de referencia interdisciplinar: el modelo argumentativo de Toulmin (2007[1958]) y la teoría del blending, de Fauconnier y Turner (1994, 1996, 1998, 2002), es decir, un cruce teórico entre dos enfoques lingüísticos que se han desarrollado sin suficiente diálogo: el lógico-factual y el cognitivo. Este trabajo atiende a esa necesidad de diálogo interdisciplinar desde la compatibilidad de las propuestas teóricas puestas en práctica.

Pese a las críticas que pesan sobre el modelo de Toulmin desde la lógica informal, específicamente las referidas a sus limitaciones para la comprensión de los argumentos en interacción social y su insuficiente separación de la lógica formal deductiva, en este texto rescatamos una perspectiva retórica del modelo en cuestión, que lo lee en función de su utilidad para "descubrir o encontrar razones que pueden permitir persuadir, convencer o llegar a acuerdos con otras personas" (Harada, 2009: 50), es decir, su consideración del problema de la aceptabilidad del argumento, manifestada en la pregunta por los medios que se utilizan para justificar las creencias que quedan implícitas en los argumentos como productos (es en este sentido que Toulmin (2007) parece concebir epistemológicamente su propuesta y presentarla como lógica práctica, aplicada o factual). 
El modelo de Toulmin pretende superar el clásico aristotélico que concebía un argumento tripartito (premisa mayor, premisa menor y conclusión), añadiendo las categorías de Garantía, Apoyo, Condiciones de refutación y Cualificadores modales, así:

"Dada nuestra experiencia general del campo en cuestión (apoyo), y de acuerdo con las reglas o principios resultantes de tal experiencia (garantía), utilizando los siguientes hechos específicos (datos), de una forma cualificada (cualificador modal), se permite concluir lo siguiente (conclusión o pretensión), a menos que exista una específica condición de refutación (excepciones)" (Santibáñez, 2009: 252).

La consideración del argumento como acontecimiento situado y producido a partir de unas condiciones siempre particulares y cambiantes, hace que el modelo de Toulmin pueda leerse más allá de lo meramente proposicional o de la artificialidad que impera en todo modelo. Nos interesa especialmente la categoría de los apoyos, que evidentemente está relacionada con los topoï aristotélicos, y que se ubica en la base del razonamiento inferencial de los argumentos, al constituir respaldos empíricos en forma de supuestos, creencias, lugares comunes, dominios conceptuales, etc., fuertemente enraizados en el conjunto social, o en palabras de Toulmin, Riecke y Janik (1979: 57): "generalizations making explicit the body of experience relied on to establish the trustworthiness of the ways of arguing applied in any particular case".

De acuerdo con Santibáñez (2009), una materialización de los apoyos lo constituye la metáfora conceptual en el engranaje argumental, siempre que se les observe como evidencia social de contenidos tácitos que estructuran dominios y comunidades discursivas. Los postulados fundamentales de la teoría de la metáfora conceptual (Lakoff, 1987, 1991, 1993, 2004; Lakoff \& Johnson, 1980, 1999) se resumen en: 1) El sistema conceptual tiene carácter metafórico inconsciente; 2) La metáfora es un modo de razonamiento sistemático orientado por la experiencia; y 3) estos sistemas funcionan sobre la base de correspondencias o proyecciones entre dominios de las experiencias humanas.

El tercer punto de esta base teórica de la metáfora conceptual es un antecedente del desarrollo de los trabajos de Fauconnier (1985) sobre los espacios mentales, en principio, y posteriormente Fauconnier y Turner (1994, 1996, 1998, 2002), la presentación de la teoría del blending. Según los autores, la unidad básica de toda organización cognitiva puede definirse a través del concepto de Espacio mental:

"Mental spaces are small conceptual packets constructed as we think and talk, for purposes of local understanding and action. Mental spaces are very partial assemblies containing elements, and structured by frames and cognitive models. They are interconnected, and can be modified as thought and discourse unfold" (Fauconnier \& Turner, 1998: 139).

Los espacios mentales son estructuras conceptuales que refieren realidades posibles con base en realidades concretas que las originan; por lo tanto, contienen 
representaciones parciales y locales, de acuerdo con cada situación donde se originen. Se destaca que esta estructura de representación es parcial y temporal, y por tanto, situada. Un espacio mental es un escenario específico y dinámico que depende del conjunto más grande y estable de los dominios dentro de los cuales se inscribe (Grady, Oakley \& Coulson, 1999). Así, por la selección cognitiva que operan, los espacios mentales tienen una función moldeadora de la realidad: "es de esperar que (...) un solo elemento pueda conceptualizarse de manera distinta dependiendo del dominio en el que se encuentre" (Pascual, 2010: 6). Con ello, se establece de manera más estrecha la relación entre sentido y acción situada, reconociendo un orden de dependencia de los significados que se construyen en cada situación de enunciación.

Fauconnier y Turner (1998) proponen un proceso de establecimiento de sentidos situados como redes de integración conceptual (blending), una operación cognitiva general de base analógica, de selección de correspondencias entre espacios de entrada (inputs), y con desarrollo y obtención de nuevas estructuras de sentidos a partir de la mezcla (blend), que 'can have effect in cognition, leading us to modify the initial inputs and to change our view of the corresponding situations'. La selección de las características que conformarán los espacios de entrada siempre es estratégica y direccionada hacia el objetivo de la mezcla, por parte de quien enuncia: esto es importante para entender cómo los discursos metafóricos orientan la comprensión de las situaciones, enfocándose en ciertos aspectos -y dejando de lado otros- de los dominios, configurando 'saliencias', y por tanto, creando los sentidos requeridos en el acto (on-line).

En este marco de referencia se incluye también el trabajo de Grady et al. (1999), quienes examinan la compatibilidad de las teorías de la metáfora conceptual y del blending, y cómo la segunda se constituye en un avance de la primera, desde intereses distintos: para la teoría de la metáfora conceptual, la intención es establecer generalizaciones al respecto de las correspondencias mentales entre conceptos en el funcionamiento de las expresiones metafóricas; mientras que la teoría del blending está enfocada en los usos particulares de ciertas expresiones en situaciones comunicativas en las cuales la comprensión solo es posible como mezcla de elementos seleccionados desde espacios mentales distintos, con lo cual se remite a los usos metafóricos, aunque no exclusivamente.

\subsection{Metodología}

Se examinan los recursos metafóricos que se presentan en 6 discursos del Presidente Juan Manuel Santos, de septiembre 26 de 2010 a julio 20 de 2011, enlistados a continuación:

- Septiembre 26 de 2010: Agradecimiento a las Fuerzas Armadas en La Macarena

- Noviembre 03 de 2010: Graduación del Curso de Altos Estudios Militares 
- Febrero 17 de 2011: Foro Ideológico del Partido Conservador

- Marzo 20 de 2011: Asamblea de la Cámara de Comercio

- Mayo 06 de 2011: Asamblea General de Proantioquia

- Julio 20 de 2011: Instalación de la Legislatura del Congreso de la República

En estos discursos se reconocieron 20 secuencias textuales en las que fue utilizada y repetida una misma metáfora, denominada para el análisis como 'metáfora de la madriguera', a través de 9 usos directos y 11 de refuerzo. Los criterios de selección de esta metáfora fueron los de recurrencia (presencia reiterativa de la metáfora en los discursos), distribución (apariciones periódicas que permitieron aplicar un muestreo sistemático bimestral) y temática bélica (usos metafóricos referidos al tema de la confrontación armada con la guerrilla de las Farc-Ep). Además de ello, se tuvieron en cuenta las condiciones de oficialidad de los comunicados y difusión mediática de los mismos, por lo cual se los extrajo de la página web de la Presidencia de la República².

Para el análisis se utilizaron las técnicas de ubicación de co-textos de los usos textuales para visualizar la ocurrencia de las unidades analizadas, y graficación procesual del funcionamiento de los conceptos y sus relaciones a medida que se realiza la indagación, tanto del blending -según lo sugieren sus mismos autores (Fauconnier \& Turner, 1998) - como del esquema argumentativo de Toulmin.

\section{Resultados}

\subsection{De guerrilleros y madrigueras}

El uso de la palabra madriguera, la 'guarida pequeña de animales salvajes' (Moliner, 2009; RAE, 2011) tiene afinidad con términos como escondrijo, cubil, ratonera, cría y fiera (Corripio, 1985), y es común encontrarlo en su sentido de sitio retirado donde se ocultan maleantes. En el discurso presidencial, el rastreo que muestra la Tabla 1 arroja la asociación de guerrilleros con madrigueras, cada vez que entra en referencia la política de seguridad democrática del actual gobierno, y configura la metáfora conceptual 'Los guerrilleros son presas (o animales de caza)': 
Tabla 1. Rastreo de la metáfora de la madriguera en la muestra.

\begin{tabular}{|c|c|}
\hline Co-texto anterior, METÁFORA y Co-texto posterior & Fecha y Discurso \\
\hline $\begin{array}{l}\text { Esta MADRIGUERA, que era la madre de las MADRIGUERAS de esta } \\
\text { organización que lleva } 50 \text { años - un cuarto de nuestra historia de República } \\
\text { independiente, generando violencia contra nuestra democracia-, esta madre } \\
\text { de las MADRIGUERAS, fue el blanco de esta operación. }\end{array}$ & $\begin{array}{l}\text { Sep. } 26-2010 \\
\text { Agradecimiento a las } \\
\text { Fuerzas Armadas en } \\
\text { La Macarena }\end{array}$ \\
\hline $\begin{array}{l}\text { No habia otra MADRIGUERA más protegida; no había otra } \\
\text { MADRIGUERA donde hubiese más gente protegiendo a este bandido, el } \\
\text { 'Mono Jojoy'. }\end{array}$ & $\begin{array}{l}\text { Sep. } 26-2010 \\
\text { Agradecimiento a las } \\
\text { Fuerzas Armadas en } \\
\text { La Macarena }\end{array}$ \\
\hline $\begin{array}{l}\text { En la reciente Operación Sodoma, que nos permitió llegar, con toda la contundencia de } \\
\text { nuestras armas y el arrojo de nuestros hombres, a la } \\
\text { MADRIGUERA del 'Mono Jojoy'. }\end{array}$ & $\begin{array}{l}\text { Nov. 3-2010 } \\
\text { Graduación del } \\
\text { Curso de Altos } \\
\text { Estudios Militares }\end{array}$ \\
\hline $\begin{array}{l}\text { Hemos escalado esa política para sacar a muchos de esos frentes de sus } \\
\text { MADRIGUERAS. }\end{array}$ & $\begin{array}{l}\text { Feb. } 17-2011 \\
\text { Foro Ideológico del } \\
\text { Partido Conservador }\end{array}$ \\
\hline $\begin{array}{l}\text { Nuestro plan [es] ir debilitando cada vez más a estos grupos narcoterroristas, de irlos } \\
\text { sacando de sus MADRIGUERAS, exponiéndolos para golpearlos. }\end{array}$ & $\begin{array}{l}\text { Feb. } 17-2011 \\
\text { Foro Ideológico del } \\
\text { Partido Conservador }\end{array}$ \\
\hline $\begin{array}{l}\text { ¿Qué ha pasado? Que nos hemos ido a las MADRIGUERAS de ciertos sitios } \\
\text { donde nunca había llegado la Fuerza Pública. }\end{array}$ & $\begin{array}{l}\text { Marzo 30-2011 } \\
\text { Asamblea de la } \\
\text { Cámara de Comercio }\end{array}$ \\
\hline $\begin{array}{l}\text { Están poniendo minas por todos lados, atacando a los policias cuando los ven, a ver si } \\
\text { atraen nuevamente a las Fuerzas Militares y al Ejército al nivel donde ellos operan para } \\
\text { quitarlos de las MADRIGUERAS. }\end{array}$ & $\begin{array}{l}\text { Marzo 30-2011 } \\
\text { Asamblea de la } \\
\text { Cámara de Comercio }\end{array}$ \\
\hline $\begin{array}{l}\text { La guerrilla en este momento, al verse replegada en forma definitiva de ciertas áreas y } \\
\text { en una politica que estamos siguiendo con el plan de guerra en forma muy cuidadosa, de } \\
\text { meternos en los sitios, en las MADRIGUERAS tradicionales desde donde operan } \\
\text { y han operado esos grupos ilegales. }\end{array}$ & $\begin{array}{l}\text { Mayo 6-2011 } \\
\text { Asamblea General } \\
\text { de Proantioquia }\end{array}$ \\
\hline $\begin{array}{l}\text { Afinar la estrategia para bacer frente a tan insidiosa campaña de las Farc, sin aflojar un } \\
\text { momento en el esfuerzo estratégico de buscarlos en sus MADRIGUERAS. }\end{array}$ & $\begin{array}{l}\text { Julio 20-2011 } \\
\text { Instalación de la } \\
\text { Legislatura del } \\
\text { Congreso de la } \\
\text { República }\end{array}$ \\
\hline
\end{tabular}

En el análisis de los co-textos resalta la asociación guerrilla-madriguera inserta en una 'definición de la situación' ' que presenta el acorralamiento del grupo insurgente y el heroísmo de las fuerzas militares del Estado. Las secuencias textuales que van conformando la narrativa del conflicto armado funcionan bajo un esquema de acción ficcional de perseguidores y perseguidos, del cazador y la presa, roles que quedan implícitos por extensión de la metáfora, pero que funcionan en los contextos de enunciación en los que son instalados bajo una lógica bélica que no entra en discusión: 'la mejor defensa es el ataque', justificación que responde a la restricción defensiva del monopolio estatal de las armas. La periodicidad recurrente de la enunciación indica que 'sacarlos de las madrigueras' se configura como parte del programa de una política militar basada en la presentación de unos y otros actores como cazadores y presas, es decir, que marca la superioridad de los primeros y de una vez el destino de los segundos ${ }^{4}$ 
roles apuntalados en el discurso, y apoyados por otro grupo de 'metáforas de refuerzo' ('los guerrilleros son animales; los guerrilleros son fieras'; 'el país tiene rincones'). A continuación se muestran algunas expresiones de ejemplo:

- "Se defienden, y es tal el desespero -yo decía y lo he dicho muchas vecesque no hay nada más peligroso que una fiera acorralada, porque se vuelve doblemente agresiva". Febrero 17, Foro ideológico Partido Conservador.

- "Hoy seguimos buscando y combatiendo a los grupos armados ilegales hasta en el último rincón del territorio. Hemos reforzado las operaciones en las zonas críticas y estamos entrando en lo más profundo de sus retaguardias, donde nunca un soldado había puesto sus botas". Julio 20, Instalación de Legislatura.

- "En el Cauca nos fuimos para arriba, para la montaña, para el pico de la montaña que era territorio de las Farc hace 40 años. Los sacamos de ahí, están desesperados". Julio 20, Instalación de Legislatura.

- "Si ustedes hacen un recuento de lo que ha sucedido en los últimos meses, golpes a estructuras y golpes a personas claves dentro de esas estructuras, ustedes se pueden dar cuenta que esa política ha sido bastante efectiva". Mayo 6, Asamblea Proantioquia.

- "Hemos logrado arrinconar a los terroristas (...) esto nos obliga a revisar los procedimientos para cerrarles el paso a cada movimiento". Noviembre 3, Graduación de Altos Estudios militares.

- "Si están en los ríos, allá iremos; si están en las colinas, allá iremos; si están en las montañas, allá iremos; si están en los páramos por encima de los 10 mil pies de altura, allá iremos; si están en las selvas más tupidas, como fuimos allá en contra del 'Mono Jojoy' allá estaremos. Porque esta es una señal muy importante: ¡No hay rincón de nuestro territorio donde nuestras fuerzas armadas no puedan llegar!"'. Septiembre 26, Agradecimiento en La Macarena.

La expresión 'metáforas de refuerzo' alude a aquellos usos metafóricos que se insertan en el marco conceptual que instala la metáfora de la madriguera, para apoyar su sentido; estos usos son estructurantes, sistemáticos y coherentes ${ }^{5}$ dentro de la metáfora (Lakoff \& Johnson, 1980), y desde el punto de vista cognitivo, están imbricadas en el sistema conceptual integrado (Fauconnier \& Turner, 1998). Como podrá verse, la estructura de este sistema se sostiene sobre la base de correspondencias analógicas entre dominios conceptuales experienciales, la apelación a ciertos espacios mentales y la configuración de un espacio integrado, amalgamado o mezclado (blend): 
El examen de los usos metafóricos (Tabla 1) y de las metáforas de refuerzo permite expresar sintéticamente el contenido global de los enunciados, en la forma oracional (1):

(1) El ejército saca a los guerrilleros de sus madrigueras.

La predicación es comprensible y aceptable al acudir a dos dominios conceptuales directamente ligados con la experiencia de los sujetos:

(1.1) Lo que el hablante conoce / cree / ha visto / ha leído / le han dicho.../ sobre los guerrilleros.

(1.2) Lo que el hablante conoce / cree / ha visto / ha leído / le han dicho.../ sobre las madrigueras.

El subrayado indica el mundo de las experiencias del hablante, al que se apela de manera indirecta y que abarca la heterogeneidad de los sujetos, pero que precisamente por su carácter volátil, amplio y difuso, es frágil a la modelación a través de los estímulos externos, especialmente a los retóricos. En este caso, la fuerza retórica reside en la correspondencia arbitraria de los conceptos GUERRILLERO y MADRIGUERA, que establece una estructura analógica traducida en (2):

(2) GUERRILLEROS ${ }^{6}$ : [MONTAÑAS]

[ANIMALES] : MADRIGUERAS

Los corchetes encierran los conceptos que son inferidos a partir de una doble lógica: por un lado, un razonamiento asociativo horizontal en términos de marcos (Fillmore, 1982) (el significado de las expresiones hace referencia a modos en que está organizado el mundo y cuyo orden se halla implícito en la misma expresión); y por otro lado, un razonamiento analógico vertical, que permite yuxtaponer dos dominios experienciales distintos: el de la vida animal y humana.

Hasta este punto no es posible todavía hablar de metaforización en un sentido completo, sino de una yuxtaposición inicial entre dos dominios conceptuales. El paso definitivo hacia la metáfora reside en la dimensión del sujeto en forma de actor o revestido de un rol (3), como se muestra en la analogía (4):

(3) Llegar a la (Nos) hemos ido a las Sacar de sus Irlos sacando de sus Buscarlos en sus Meternos en sus Quitarlos de las madriguera

madrigueras madrigueras a los frentes madrigueras madrigueras madrigueras madrigueras 
(4) El Ejército saca a los guerrilleros de sus refugios:

El cazador saca a las presas de sus madrigueras.

Esto significa que es la intervención del sujeto en acción la que opera de manera transformadora en la analogía (2) estableciendo el nuevo complejo analógico (2’):

\section{(2’) EJÉRCITO : GUERRILLEROS : [REFUGIOS] \\ CAZADOR : [ $\underline{\text { PRESAS}}] \quad:$ MADRIGUERAS}

El paso del concepto del sistema (2) al (2’) requiere además dos precisiones: la primera atiende al tránsito ANIMALES $\rightarrow$ PRESAS, que se explica por una especificidad causal que introduce el agente en la asociación EJÉRCITO-CAZADOR (si el ejército es el cazador, entonces los guerrilleros son sus presas); la segunda, debe aclarar el tránsito MONTAÑAS $\rightarrow$ REFUGIOS, término de llegada que en principio probablemente no aparezca en el marco del concepto GUERRILLERO, pero que se propone como término integrador frente al hecho textual de que en el corpus, las madrigueras son asociadas tanto con ESCONDITES como con LUGAR DE OPERACIONES.

Pero aun aceptando (2'), sucumbir a la realización de correspondencias (universales, lineales, unidireccionales y predecibles) entre los dominios demarcados, es decir, trazar el mapping, sería reducir las particularidades de la situación específica del uso metafórico a un proceso de transmisión mecánica de dominios-fuente a dominiosmeta; en vez de esto, utilizamos la teoría del blending para rastrear la integración conceptual entre los dominios de entrada descritos en el párrafo anterior.

En el caso de la metáfora de la madriguera, los espacios de entrada y el sistema de correspondencias privilegian las categorías experienciales que se resaltan en la Figura 1:

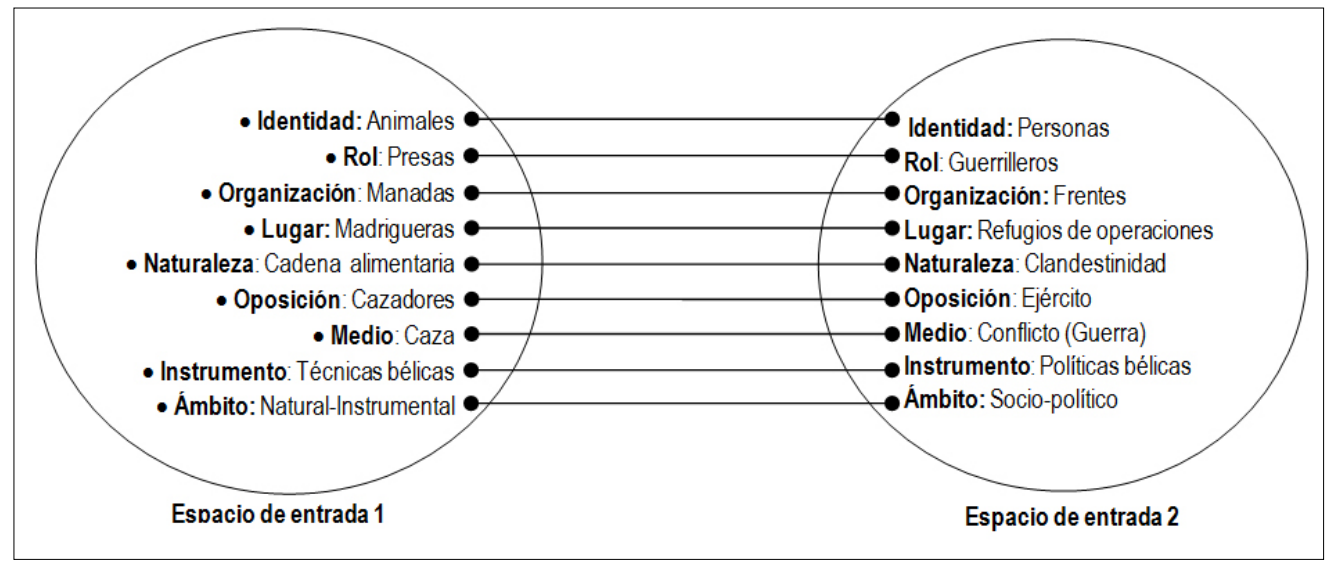

Figura 1. Espacios de entrada de la metáfora 'Los guerrilleros son presas'. 
Nótese que los espacios definidos corresponden a ciertas 'saliencias' de los dominios, pero no a su totalidad, por lo cual la situación enunciativa deja por fuera posibles características de ambos dominios, especialmente sus objetivos y metas (espacio ${ }_{1}$ : denuncia, insurgencia; espacio ${ }_{2}$ : supervivencia). Asimismo, las categorías componen una red interna en los espacios, articulada a su vez externamente con el entramado discursivo general, de tal modo que los énfasis en ellas son los que moldean la comprensión de la situación. El mecanismo analógico crea una interconexión entre los sistemas, un espacio de intersección conceptual o espacio genérico (generic space, Fauconnier \& Turner, 1998) que contiene lo que ambos espacios de entrada comparten en el desarrollo de la red de integración conceptual (Figura 2):

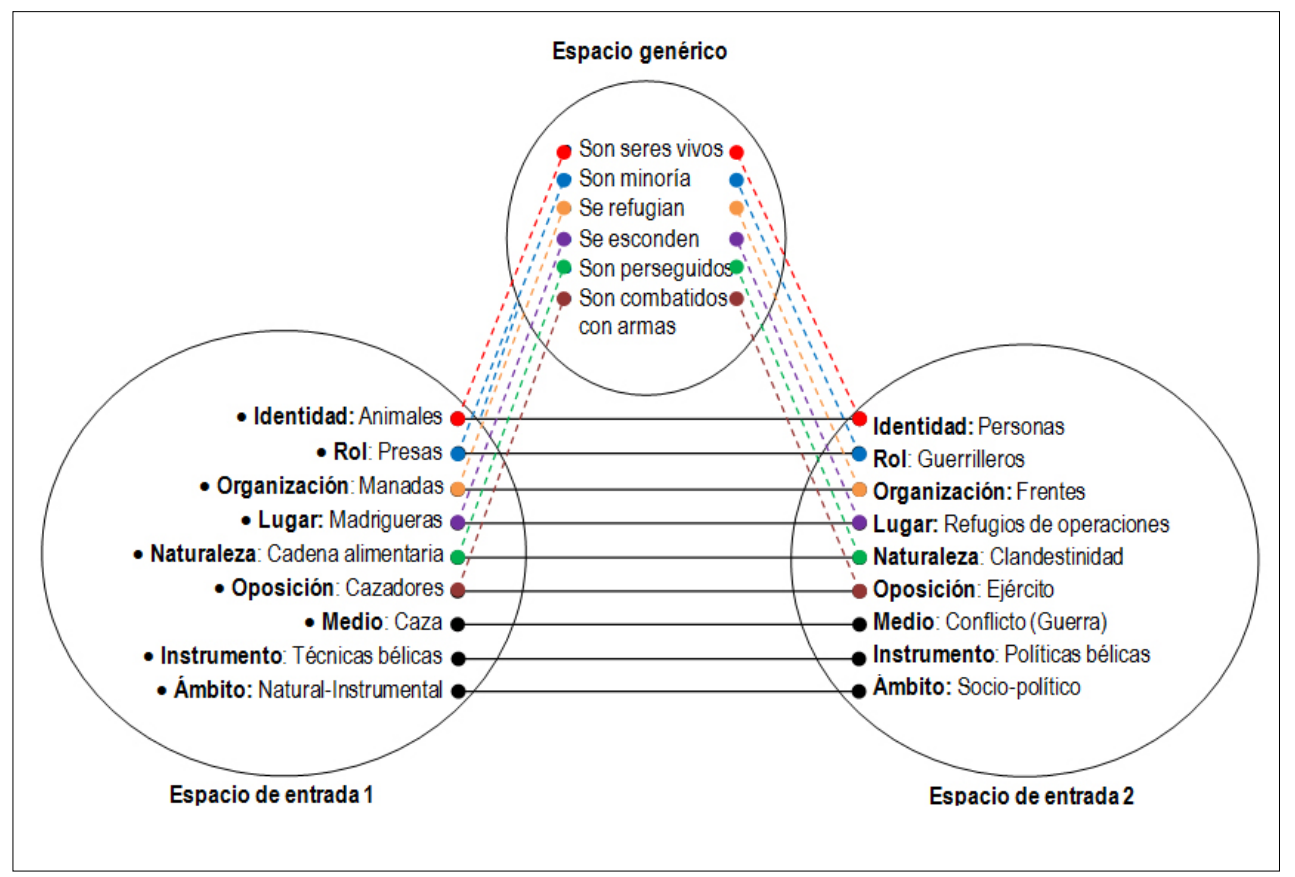

Figura 2. Proyección del espacio genérico en la metáfora 'Los guerrilleros son presas.'

El espacio genérico es una proyección de las relaciones entre los espacios de entrada, que permite despejar cómo es que se da a entender estratégicamente un elemento en términos de otro a partir de lo que ellos tienen en común. En este 'lugar' del razonamiento, el sujeto descarta aquellas expresiones que no se direccionan en el mismo sentido performativo, aunque sean posibles sus intersecciones desde la experiencia: por ejemplo, en la situación que se discute un espacio de entrada como 'árbol del bosque', en vez de 'presas', podría generar categorías experienciales similares, pero arrojaría un espacio genérico totalmente distinto (esto refuerza la idea de la integración conceptual como proceso en acto). 
La mezcla, integración, fusión o amalgama ${ }^{7}$ (blend) completa el esquema con un espacio proyectado desde los espacios de entrada, cuyo contenido es una selección de algunos de sus elementos y la aparición de una nueva estructura de sentido (emergent structure). Todos los elementos de la integración están cohesionados con las estructuras de los espacios de entrada, y el nuevo sentido es generado a través de un razonamiento inferencial (Figura 3):

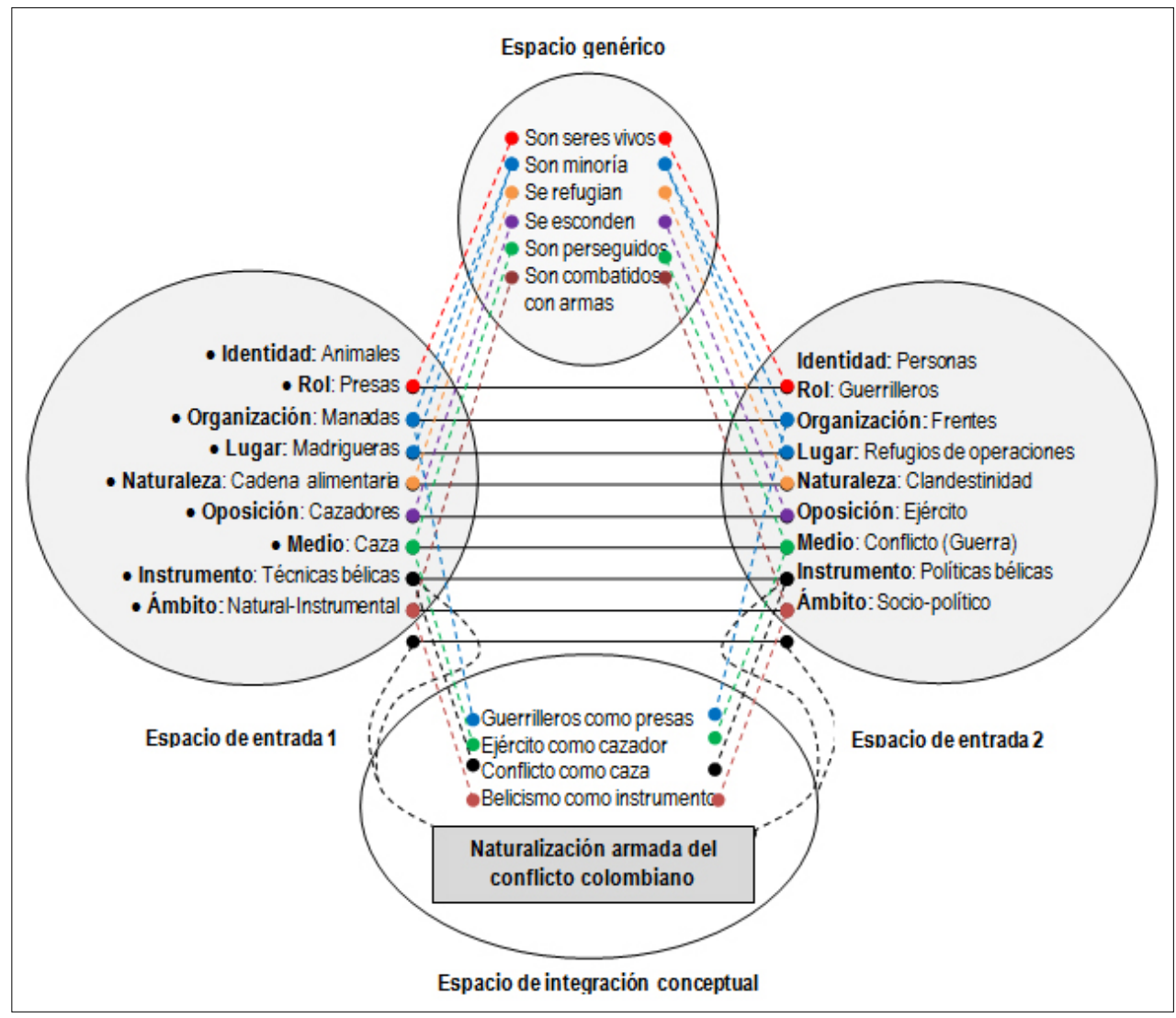

Figura 3. Red de integración conceptual de la metáfora 'Los guerrilleros son presas' (Fauconnier \& Turner, 1998). 
Grady et al. (1999) advierten que específicamente en la integración metafórica, ciertos aspectos de las entradas son ocultados y otros destacados, y la visibilidad de los primeros se pierde en la presencia del nuevo concepto integrado; así, hay información de los espacios de entrada que es ignorada en la integración (Grady et al., 1999). La relación del cazador y la presa funciona en el ámbito natural-instrumental, pero reflejarlo en la interacción humana entre guerrilleros y ejército, es un artificio direccionado. De igual manera, la instrumentalización del belicismo (de lo técnico a lo político) y la presentación del conflicto como actividad de caza, se insertan en la pretensión de naturalizar el carácter armado del conflicto.

El espacio conceptual integrado se instaura como un escenario unificado y como un sistema de sentidos 'objetivizable' (en cuanto a la posibilidad de manipularlo como un objeto de conocimiento), utilizable en otras situaciones direccionadas hacia fines análogos. La red de integración es analíticamente reversible partiendo desde el espacio conceptual integrado. El 'desempaquetamiento' o 'desembalaje' (unpacking) permite reconstruir los espacios de entrada, el genérico y las correspondencias. Además, los elementos seleccionados en la integración y la nueva estructura tienen carácter de relevancia en cuanto al direccionamiento de la acción: los roles de cazador y presa se resuelven de manera inmediata en la percepción, porque acuden a elementos que guardan relaciones estrechas con la experiencia de los sujetos. El fenómeno del conflicto no es definido en la dimensión de su complejidad, sino de la simpleza que reside en la analogía de la caza. Pascual (2010) sintetiza estas operaciones en cuatro objetivos pragmáticos:

1. Comprimir lo que es difuso (el conflicto armado presentado como caza).

2. Obtener una visión global (insertar el fenómeno dentro de una política).

3. Contar una historia (narrativizar el hecho).

4. Hacer inducción (orientar la extrapolación del caso al comportamiento general).

Con el análisis se evidencia que más allá de las relaciones metafóricas convencionales (de un dominio-fuente a un dominio-meta), estas pueden ser el punto de partida de procesos más complejos donde emergen espacios conceptuales integrados. Estos espacios contienen dimensión argumentativa y, a fuerza del uso, van borrando los nexos implícitos y artificiales entre los conceptos e instaurando los sentidos emergentes, con sus respectivas implicaciones en forma de estructuras integradas:

Donde X es ‘Guerrilleros'; Y es 'presas'; Z es ‘acción bélica’:
(1) dado que $\mathrm{X}$ es $\mathrm{Y}$, entonces Z;
(2) dado que $\mathrm{X}$ es $\mathrm{Y}$, es razonable que Z;
(3) dado que $\mathrm{X}$ es $\mathrm{Y}$, es necesario Z;
(4) dado que X es Y, se legitima Z. 
Mientras que las estructuras (1) y (2) funcionan de manera causal bajo el razonamiento lógico clásico, (3) y (4) se inclinan más por un funcionamiento de problema-solución no necesariamente deductivo, ni inductivo, ni siquiera abductivo. $Z$ es tanto proceso como producto, y está expresada cuidadosamente en usos indirectos como 'Operación', 'Estrategia', 'Esfuerzo', 'Política', 'Arrinconar', 'uso contundente de las armas' 'Ir debilitando', 'Ir sacando' e 'Irnos metiendo en las madrigueras'. Se percibe también que la referencia a los ataques armados siempre está presentada como la reacción a una acción primera que la provoca, índice de un proyecto de justificación que subyace al discurso y que vertebra la narración de las acciones en función del despliegue de argumentos. Funcionando como sistema, el recorrido desde (1) hasta (4) es la huella de los pasos que sigue el razonamiento formal hacia una lógica informal desplegada desde el discurso, una lógica de la acción, mediada por la metáfora y orientada hacia la auto-legitimación a partir de la deslegitimación del otro.

\section{Metáfora y legitimación}

La legitimación es un motor de la acción y de la estabilidad de las políticas públicas; es el resultado de la lucha por la legitimidad del comportamiento de los actores públicos, la justificación de las relaciones de poder y de los paradigmas dominantes (Cruz-Rubio, 2010), para Boltanski y Chiapello (2002), tal legitimación se construye sobre la base de sistemas de principios y valores dentro de los cuales se despliegan argumentos que ordenan status quos (regímenes de justificación). En el caso de la política belicista extendida en el discurso, hay una búsqueda de legitimación del uso de la violencia que rebasa la referencia al monopolio estatal de las armas y acude a la presentación del Otro como minoría natural, presa o animal de caza para amortiguar la contradicción ética consustancial (combatir la violencia con violencia), reforzar la imagen del agresor como categoría inamovible o etiqueta y menos como rol intercambiable entre los actores, y conseguir el apoyo del tercero (la ciudadanía) en forma de opinión pública. Sabucedo et al. (2004) ven en este último punto una clara finalidad persuasiva de los discursos de legitimación.

El recurso metafórico por parte del Estado no solo se orienta hacia la deslegitimación de la guerrilla (para lo cual otras estrategias más directas resultan también efectivas, como la demonización y la rotulación terrorista), sino también hacia una doble autolegitimación: 1) de la fuerza militar, en virtud de las acusaciones que pesan sobre estas instituciones, provocadas a raíz de incidentes históricos de crímenes de Estado, tanto en el pasado lejano como reciente; y 2) de la política militarista, en respuesta a los sectores de la población y a los internacionales que consideran obligación del gobierno la iniciativa de la salida pacífica del conflicto. En esta medida, la metáfora adquiere dimensión persuasiva al presentar la violencia como reacción, necesidad y heroicidad, núcleo de una estrategia política legítima y en el marco de narrativas que simplifican el conflicto a la caricatura del cazador y la presa. 
Siguiendo a Santibáñez (2009), es posible ubicar las metáforas conceptuales en un 'engranaje argumental' que vincule el estudio de la metáfora con las indagaciones teóricas sobre la argumentación. El autor propone un marco metodológico basado en el modelo de correspondencias entre dominios (Lakoff \& Johnson, 1980; Lakoff \& Turner, 1989) por medio del cual se rastrea el mapeo a partir de la identificación y caracterización aspectual de los dominios, la descripción de la lógica situacional y la determinación de la metáfora conceptual por inferencia. Esta reconstrucción es presentada bajo la lógica factual del modelo argumentativo de Toulmin (1958), como se aplica en la Figura 4:

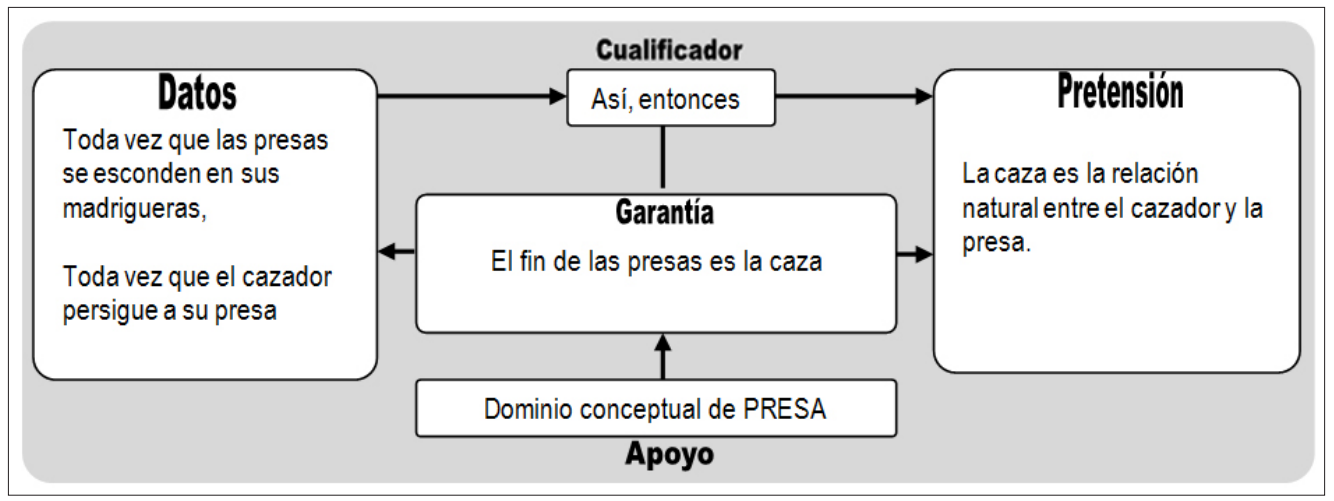

Figura 4. Metáfora como argumento en el esquema de Toulmin (1958) (Basado en Santibáñez, 2009).

En la Figura 4 se propone abrir la analogía contenida en la metáfora y atender primero a la información restringida a uno de los espacios de entrada: el de la presa. El paso de los datos a la pretensión se sostiene sobre la garantía (principio general o norma tácita formal) según la cual el destino de las presas es la caza; a su vez,tal garantía se respalda en un apoyo (mundo sustancial) de carácter definitorio, que corresponde al dominio conceptual del significado de PRESA: "animal que puede ser cazado por otro animal o por el hombre" (RAE, 2011). En la Figura 5 se hace lo propio con la información del segundo espacio de entrada: el de los guerrilleros: 


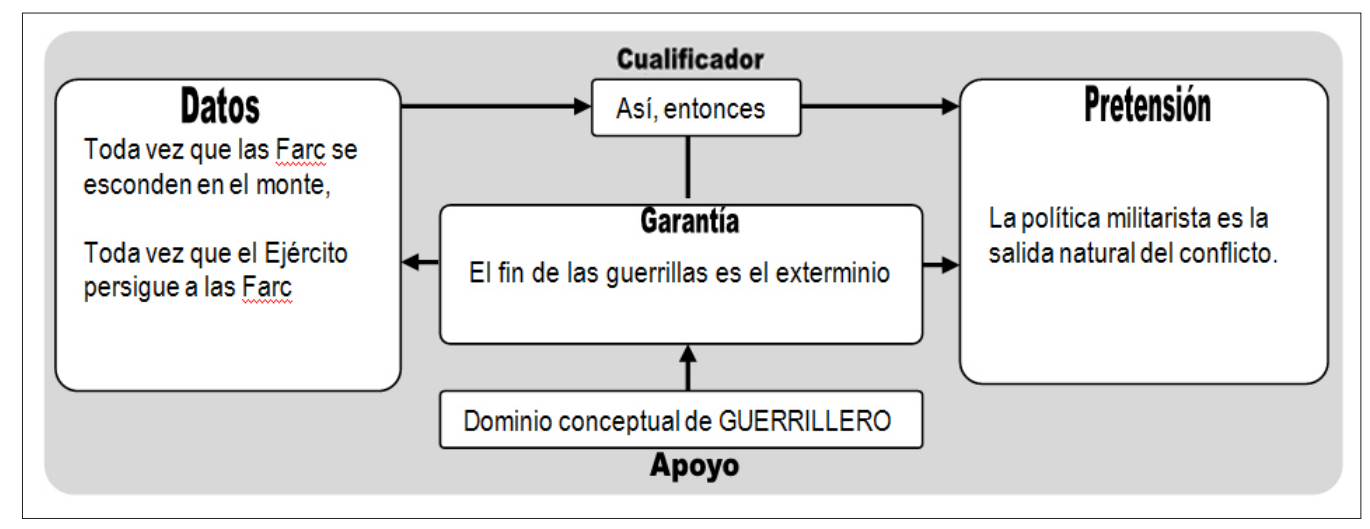

Figura 5. Metáfora como argumento en el esquema de Toulmin (1958, 1979) (Basado en Santibáñez, 2009).

En la Figura 5 se abarca la segunda parte de la analogía para poner en evidencia que la pretensión está sostenida sobre una garantía falaz de generalización apresurada: 'algunas guerrillas han sido exterminadas, por lo tanto, toda guerrilla verá tal fin'. En este caso, la garantía se apoya también sobre el dominio conceptual del término GUERRILLERO, pero específicamente en la inscripción de este término dentro de un marco de interpretación cognitiva que lo supedita a los conceptos de terrorismo y narco-terrorismo, como se ve explícitamente en el corpus, y como ha sido examinado en otros trabajos sobre la configuración de este marco (Chomsky, 2000; Lakoff, 2004; Rosell, 2009; Vega, 2009; Olave, 2011).

Al abrir la metáfora para analizarla se comprende cómo la pretensión (2) de la Figura 5, sostenida sobre una garantía falaz y un apoyo construido discursivamente, refuerza su contundencia persuasiva al imbricarse con el sistema argumentativo (1) de la Figura 4. Se comprende aquí que acontece una integración conceptual de la cual emerge una estructura combinada nueva, desde la teoría del blending, y que tal integración se puede presentar también siguiendo el modelo argumentativo de Toulmin (1958), guardando consistencia y coherencia con la Figura 4, pero enfocándose en este caso en la dimensión argumentativa de tal integración conceptual (Figura 6). 


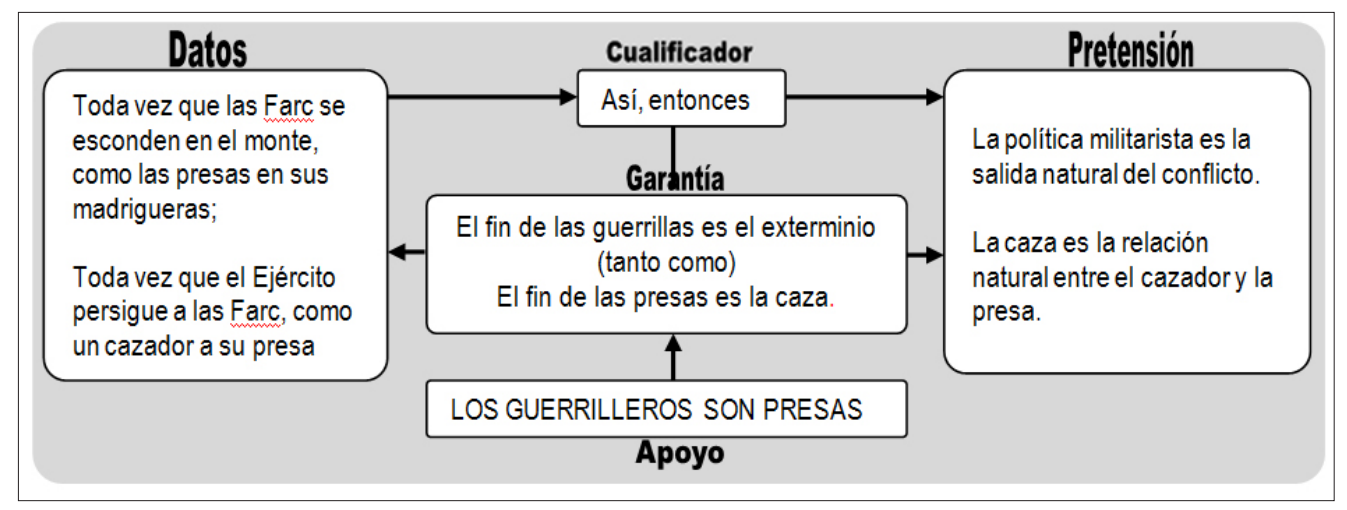

Figura 6. Metáfora como argumento en el esquema de Toulmin $(1958,1979)$ (Basado en Santibáñez, 2009).

En la Figura 6 la metáfora conceptual cumple la función del apoyo. De acuerdo con la lingüística cognitiva, Santibáñez (2009: 259) argumenta que ellas "funcionan como experiencia, esto es, nacen como producto de la experiencia que genera, en el transcurso del tiempo, sentido y comprensión de lo que rodea”, y además de ello, cita la definición de Apoyo en Toulmin et al. (1979: 57): "generalizations making explicit the body of experience relied on to establish the trustworthiness of the ways of arguing applied in any particular case". Ambas definiciones hacen plausible el lugar de las metáforas conceptuales en el apoyo. Así presentado, el esquema permite organizar la metáfora conceptual en clave de argumento, mientras que al presentarlo utilizando el modelo de Fauconnier \& Turner (1998), mostrado en la Figura 3, la metáfora conceptual queda comprendida como proceso cognitivo.

\section{CONCLUSIONES}

Disponiendo frente a frente las aplicaciones de ambos modelos, queda en evidencia la relación coherente entre el espacio de integración, en el blending, y la pretensión en el esquema de Toulmin. Teniendo en cuenta que este atiende más al modelo de correspondencias entre dominios, de Lakoff y Johnson (1980) y Lakoff y Turner (1989), es posible asumir como complementarios los modelos, al centrar su interés en diferentes aspectos de los procesos de pensamiento involucrados en el decir argumentativo; así lo sugieren Grady et al. (1999: 121):

"The conventional conceptual pairings and one-way mappings studied within Conceptual Metaphor Theory are inputs to and constraints on the kinds of dynamic conceptual networks posited within Blending Theory". 
La mayor dificultad al utilizar ambos modelos para aproximarse al estudio de un caso reside, entonces, en olvidar que cada uno de ellos tiene intereses parciales y énfasis particulares; de lo contrario, el análisis resulta enriquecido.

El análisis ejemplifica cómo la metáfora conceptual establece un marco cognitivo dentro del cual es difícil que la audiencia ponga en discusión la aceptabilidad, relevancia y adecuación de las premisas. Tanto el apoyo como la garantía, o lo que es igual, tanto la fuerza de las experiencias como la fuerza de los principios, se presentan como construcciones discursivas que refuerzan la aceptabilidad de la pretensión, entendiendo esta última como un espacio conceptual integrado. Así, un campo crítico que queda pendiente es la evaluación más detenida del argumento cuando la metáfora conceptual se encuentra en el apoyo y sostiene una garantía falaz, como sucede en el caso estudiado de la metáfora de la madriguera. Esto nos lleva a advertir, con Santibáñez (2009: 266), que:

"Si el análisis metafórico cognitivista quiere tener éxito desde una
perspectiva de la argumentación, debe ser incorporado a una teoría que
conciba el uso de metáforas como procedimientos sujetos a evaluación
crítica, distinguiendo, cuando sea oportuno, cuándo y por qué el uso de
una metáfora conceptual deviene en falacia".

El estudio de los usos argumentativos de la metáfora todavía está fuertemente marcado por la dicotomía persuasión-argumentación que los trabajos contemporáneos en el campo han vuelto a pensar como difícilmente desligables, no excluyentes y en formato de continuum. Habría que preguntarse, con Tindale (2004), si el énfasis de una teoría argumentativa no debería estar más bien en las formas a través de las cuales, en la vida cotidiana, consideramos aceptables ciertas pretensiones, al margen de la lógica deductiva formal, y dentro del carácter dialógico del discurso. En este sentido, metáfora y legitimación aparecen fuertemente ligadas a las acciones políticas, en clave de cognición social.

Para el caso colombiano, la simplificación de la dimensión discursiva del conflicto armado opera de manera inversa a los intereses de la teoría sobre el conflicto, pues desenfoca las raíces culturales que le permiten permanecer instalado en los modos de ser de sus actores y obvia la construcción sociocognitiva e intersubjetiva de la realidad; construcción que utiliza recursos como la metáfora conceptual para presentar el belicismo, muchas veces, como coherente, lógico e inevitable, es decir, que instaura unos imaginarios sociales legitimadores.

Pero una instauración tal no es una implantación simple y mecánica de quien enuncia, ni el producto de una dialéctica basada en el despliegue de argumentos razonados, sino un complejo proceso de interacción cuyo funcionamiento todavía está lejos de ser integralmente descrito. 


\section{REFERENCIAS BIBLIOGRÁFICAS}

Boltanski, L. \& Chiapello, E. (2002). El nuevo espíritu del capitalismo. Madrid: Akal.

Castro Caycedo, G. \& CMP (Corporación Medios para la Paz) (2005). Para desarmar la palabra. Diccionario de términos del conflicto y de la paz. Bogotá: CMP.

Chihu, A. (2002). El análisis cultural de los movimientos sociales. Revista Sociológica, 42, 209-230.

Chomsky, N. (2000). La moralidad distorsionada. ¿Guerra al terrorismo? Conferencia en la Universidad de Harvard [en línea]. Disponible en: http://es.scribd.com/ doc/15682584/chomsky-noam-moralidad-distorsionada-pdf

Corripio, F. (1985). Diccionario de ideas afines. Madrid: Herder.

Cruz-Rubio, C. (2010). Legitimación y regímenes de politica pública: Hacia una mejor comprensión de la estabilidad y el cambio [en línea]. Disponible en: http://ortegaygasset. academia.edu/cesarcruzrubio/papers/153396

Entman, R. (1993). Framing. Journal of communication, 43(4), 51-58.

Estrada, F. (2000). El lenguaje de la guerra y la política en Colombia. Reflexión Politica, 2(4), 1-8.

Estrada, F. (2004). Las metáforas de una guerra perpetua. Estudios sobre pragmática del discurso en el conflicto armado colombiano. Medellín: Fondo Editorial Universidad Eafit.

Fauconnier, G. (1985). Mental spaces. Cambridge: MIT Press.

Fauconnier, G. \& Turner, M. (1994). Conceptual projection and middle spaces [en línea]. Disponible en: http:// cogsci.ucsd.edu

Fauconnier, G. \& Turner, M. (1996). Blending as a central process of grammar. En A. Goldberg (Ed.), Conceptual structure, discourse and language (pp. 113-129). Stanford: Center for the Study of Language and Information.

Fauconnier, G. \& Turner, M. (1998). Conceptual integration networks. Cognitive Science, 22(2), 133-187.

Fauconnier, G. \& Turner, M. (2002). The way we think. Nueva York: Basic Books.

Fillmore, C. (1982). Frame semantics. En Linguistic Society of Korea (Ed.), Linguistics in the morning calm (pp. 111-137). Seúl: Hanshin.

Goffman, E. (1974). Frame analysis: An essay on the organization of experience. Londres: Harper and Row. 
Gómez, O. (2009). Secuestro en Colombia: Significados y prácticas discursivas. Tesis de magíster, Universidad Nacional de Colombia, Bogotá, Colombia.

Grady, J., Oakley, T. \& Coulson, S. (1999). Blending and metaphore. En G. Steen \& R. Gibbs (Eds.), Metaphor in cognitive linguistics (pp. 101-124). Amsterdam/ Philadelphia: John Benjamin.

Harada, E. (2009). Algunas aclaraciones sobre el 'modelo' argumentativo de Toulmin [en línea]. Disponible en: http://www.izt.uam.mx/contactos/n73ne/toulmin.pdf

Lakoff, G. (1987). Women, fire and dangerous things. Chicago: University of Chicago Press.

Lakoff, G. (1991). La metáfora en politica. Carta abierta a Internet [en línea]. Disponible en: http://serbal.pntic.mec.es/ cmunoz11/guerra.pdf

Lakoff, G. (1993). The contemporary theory of metaphor. En A. Ortony (Ed.), Metaphor and thought (pp. 202-251). Cambridge: Cambridge University Press.

Lakoff, G. (2004). No pienses en un elefante. Madrid: Editorial Complutense de Madrid.

Lakoff, G. \& Johnson, M. (1980). Metáforas de la vida cotidiana. Madrid: Cátedra.

Lakoff, G. \& Johnson, M. (1999). Philosophy in the flesh, the embodied mind and its challenge to western thought. Nueva York: Basic Books.

Lakoff, G. \& Turner, M. (1989). More than cool reason. Chicago: University of Chicago Press.

Moliner, M. (2009). Diccionario de uso del español. Madrid: Gredos.

Olave, G. (2011). Los marcos del conflicto. Aproximación al conflicto armado colombiano desde el concepto de marco. Discurso \& Sociedad, 5(3), 514-546.

Pascual, C. (2010). Los espacios mentales y la integración conceptual [en línea]. Disponible en: http://www.rug.nl/staff/e.pascual/espaciosmentales.pdf

Posada Carbó, E. (2002). ¿Guerra civil? El lenguaje del conflicto en Colombia. Bogotá: Alfaomega, Fundación Ideas para la Paz.

Posada Carbó, E. (2003). El lenguaje del presidente Uribe frente al terrorismo [en línea]. Disponible en: http://www.ideaspaz.org/articulos/download/

Posada Carbó, E. (2005). Lenguaje, poder y políticas de paz [en línea]. Disponible en: http://www.ideaspaz.org/articulos/download/

Posada Carbó, E. (2006). El lenguaje presidencial y las perspectivas de paz [en línea]. Disponible en: http://www.ideaspaz.org/articulos/download/ 
RAE (Real Academia Española) (2011). Diccionario virtual RAE [en línea]. Disponible en: http:// rae.es

Rosell, C. (2009). El poder de la palabra. Un análisis de 21 extractos del discurso presidencial colombiano con respecto a las FARC. Tesis de magíster, Universidad de Lund, Lund, Suecia.

Sabucedo, J., Barreto, I., Borja, H., De la Corte, L., Durán, M. \& Blanco, A. (2004). Deslegitimación del adversario y violencia política: El caso de las FARC y las AUC en Colombia. Acta Colombiana de Psicología, 12, 69-85.

Sabucedo, J., Barreto, I., Borja, H., De la Corte, L. \& Durán, M. (2006). Legitimación de la violencia y contexto. Análisis del discurso de las FARC-EP. Estudios de Psicología, 27, 279-292.

Sabucedo, J., Barreto, I., Borja, H. \& López, W. (2008). Construcción del discurso deslegitimador del adversario: Gobierno y paramilitarismo en Colombia [en línea]. Disponible en: http://pepsic.bvsalud.org/scielo.php?pid=s165792672008000200020\&script =sci_arttext

Salamanca, L. (2006). Entre balas y palabras. Relaciones discursivas en torno al conflicto armado colombiano [en línea]. Disponible en: http://halshs.archives-ouvertes.fr/ halshs-00104038/

Santibáñez, C. (2009). Metáforas y argumentación: Lugar y función de las metáforas conceptuales en la actividad argumentativa. Revista Signos. Estudios de Lingüistica, 42(70), 245-269.

Shlenker, J. \& Iturralde, M. (2006). El uso del discurso de los derechos humanos por parte de los actores armados en Colombia: ¿Humanización del conflicto o estrategia de guerra? Análisis Político, 1(56), 29-50.

Thomas, W. (1972). The definition of situation. En J. Manis \& B. Meltzer (Eds.), Symbolic interaction (pp. 331-336). Boston: Allyn and Bacon, Inc.

Tindale, C. (2004). Rhetorical argumentation. Principles of theory and practice. California: Sage.

Toulmin, S. (2007[1958]). Los usos de la argumentación. Madrid: Península.

Toulmin, S., Rieke, R. \& Janik, A. (1979). An introduction to reasoning. Nueva York: Macmillan Publishing Co., Inc.

Vega, J. (2009). Análisis del conflicto armado colombiano como creador de imaginarios colectivos para la sociedad durante el período Pastrana y Uribe. Tesis de magíster, Universidad del Rosario, Bogotá, Colombia.

Vizcaíno, M. (2004). Secuestrados o prisioneros de guerra: Visiones de un mismo conflicto armado. Medellín: Universidad Cooperativa de Colombia. 


\section{NOTAS}

1 Este documento forma parte del proyecto de tesis doctoral titulado 'La construcción retórica del conflicto armado colombiano', del Doctorado en Letras de la Universidad de Buenos Aires, proyecto dirigido por la Doctora Elvira Narvaja de Arnoux.

2 http://www.presidencia.gov.co/Discursos

3 Este concepto es analizado, entre otros, por Entman (1993) y Chihu (2002) desde el análisis de marcos en sociología, y sus referentes de base son Thomas (1972), quien acuña el concepto, y Goffman (1974), con la teoría de los marcos de interpretación.

4 El discurso del presidente Santos también caracteriza por la estrategia de la intimidación verbal dentro de este programa contrainsurgente, en secuencias como "mi mensaje nuevamente, como lo hice hace tres años, a las Farc, es no continúen con esa lucha. Esa lucha no los va a llevar a ningún lado. Los va a llevar a la tumba, eso sí, o a la cárcel" (Discurso de marzo 1 de 2011 [en línea]. Disponible en: http://wsp.presidencia.gov.co/Prensa/2011/Marzo).

5 Lakoff y Johnson (1980: 136) señalan que "las implicaciones metafóricas desempeñan un papel esencial en la unión de todos los ejemplos de una sola estructuración metafórica de un concepto".

6 Así como / Tal como / Del mismo modo que /

7 El escaso número de traducciones al español de las obras teóricas principales ha diseminado una terminología muy inestable. 